\title{
Statistical Korovkin and Voronovskaya type theorem for the Cesáro second-order operator of fuzzy numbers
}

\author{
Naim L. Braha and Valdete Loku
}

\begin{abstract}
In this paper we define the Cesáro second-order summability method for fuzzy numbers and prove Korovkin type theorem, then as the application of it, we prove the rate of convergence. In the last section, we prove the kind of Voronovskaya type theorem and give some concluding remarks related to the obtained results.
\end{abstract}

Mathematics Subject Classification (2010): 40A10, 40C10, 40E05, 40A05, 40G99, $26 \mathrm{E} 50$.

Keywords: Cesáro second order summability method, statistical convergence, Korovkin type theorem, rate of convergence, Voronovskaya type theorem.

\section{Introduction}

The concepts of fuzzy sets and fuzzy set operations were first introduced by Zadeh [16] and subsequently, several authors have discussed various aspects of the theory and applications of fuzzy sets such as fuzzy topological spaces, similarity relations, and fuzzy orderings, fuzzy measures of fuzzy events, fuzzy mathematical programming. Matloka [13] introduced bounded and convergent sequences of fuzzy numbers studied some of their properties and showed that every convergent sequence of fuzzy numbers is bounded.

In the present paper, we will prove the Korovkin type theorem for statistical summability $(C, 2)$ and the rate of convergence. In this section, we give a brief overview of statistical convergence, fuzzy numbers, and sequences of fuzzy numbers. In section 2 we prove the main results of this paper. In section 3 we give results related to the rate of convergence.

The idea of statistical convergence depends upon the density of subsets of the set $\mathbb{N}$ of natural numbers. We shall denote by $\mathbb{N}$ the set of all natural numbers. Let $K \in \mathbb{N}$ and $K_{n}=\{k \leq n: k \in K\}$. Then the natural density of $K$ is defined by 
$d(K)=\lim _{n \rightarrow \infty} \frac{\left|K_{n}\right|}{n}$ if the limit exists, where the vertical bars indicate the number of elements in the enclosed set. The sequence $x=\left(x_{k}\right)$ is said to be statistically convergent to $L([10])$ if for every $\varepsilon>0$, the set $K_{\varepsilon}=\left\{k \in \mathbb{N}:\left|x_{k}-L\right| \geq \varepsilon\right\}$ has natural density zero, i.e. for each $\varepsilon>0$,

$$
\lim _{n \rightarrow \infty} \frac{1}{n}\left|\left\{k \leq n:\left|x_{k}-L\right| \geq \varepsilon\right\}\right|=0 .
$$

In this case, we write $s t-\lim x=L$. Note that every convergent sequence is statistically convergent but not conversely.

In paper [6], was defined the second order Cesáro summability method as follows:

$$
(C, 2)_{n}=\sum_{n=1}^{\infty}\left(\frac{1}{(n+1)(n+2)} \sum_{k=0}^{n}(n+1-k)\left|x_{k}\right|\right) .
$$

The summability method $(C, 2)_{n}$ is a regular. We say that the series $\sum_{n=1}^{\infty} x_{n}$ is $(C, 2)_{n}-$ summable to $L$ if

$$
\lim _{n} \sum_{j=1}^{n}\left(\frac{1}{(j+1)(j+2)} \sum_{k=0}^{j}(j+1-k)\left|x_{k}\right|\right)=L .
$$

In the present paper, we define Cesáro second-order summability method for sequences of fuzzy numbers and give Korovkin type theorem and rate of convergence. The theory of Korovkin type theorems was intensively investigated in recent years, see for example $[3,4,1,6,7,8,9,11,12]$.

\section{Preliminaries}

Let $C(\mathbb{R})$ denote the family of all nonempty, compact, convex subsets of $\mathbb{R}$. Denote by

$$
L(\mathbb{R})=\{u: \mathbb{R} \rightarrow[0,1]: u \quad \text { satisfies } \quad(1)-(4) \text { bellow }\}
$$

where

1. $u$ is normal, there exists an $x_{0} \in \mathbb{R}$ such that $u\left(x_{0}\right)=1$,

2. $u$ is fuzzy convex, for any $x, y \in \mathbb{R}$ and $0 \leq \lambda \leq 1, u(\lambda x+(1-\lambda) y) \geq$ $\min [u(x), u(y)]$,

3. $u$ is upper semicontinuous,

4. the closure of $\{x \in \mathbb{R}: u(x)>0\}$, denoted by $[u]_{0}$, is compact.

If $u \in L(\mathbb{R})$, then $u$ is called fuzzy number, and $L(\mathbb{R})$ is said to be fuzzy number space. For $0<\alpha \leq 1$, the $\alpha$ - level set $[u]_{\alpha}$ of $u$ is defined by $[u]_{\alpha}=\{x \in \mathbb{R}: u(x) \geq \alpha\}$. Then from (1)-(4), it follows that the $\alpha$-level sets $[u]_{\alpha} \in C(\mathbb{R})$.

The set of real numbers can be embedded in $L(\mathbb{R})$, since each $r \in \mathbb{R}$ can be regarded as a fuzzy number $\bar{r}$ defined by

$$
\bar{r}= \begin{cases}1 ; & \text { if } \quad x=r \\ 0 ; & \text { if } \quad x \neq r\end{cases}
$$


Let $u, v, w \in L(\mathbb{R})$ and $k \in \mathbb{R}$. Then the operations addition and scalar multiplications are defined in $L(\mathbb{R})$ as follows:

$$
\begin{gathered}
u+v=w \Leftrightarrow[w]_{\alpha}=[u]_{\alpha}+[v]_{\alpha} \text { for all } \alpha \in[0,1], \\
\Leftrightarrow w_{\alpha}^{-}=u_{\alpha}^{-}+v_{\alpha}^{-} \text {and } w_{\alpha}^{+}=u_{\alpha}^{+}+v_{\alpha}^{+} \text {for all } \alpha \in[0,1], \\
{[k u]_{\alpha}=k[u]_{\alpha} \text { for all } \alpha \in[0,1] .}
\end{gathered}
$$

Further details related to the structural properties of the fuzzy numbers, are given in [5]. Let us denote by $W$ the set of all closed bounded intervals $A$ of real numbers with endpoints $\underline{A}$ and $\bar{A}$, i.e., $A=[\underline{A}, \bar{A}]$. Define the relation $d$ on $W$ by

$$
d(A, B)=\max \{|\underline{A}-\underline{B}|,|\bar{A}-\bar{B}|\} .
$$

Then it can be easily observed that $d$ is a metric on $W$ and $(W, d)$ is a complete metric space, $([14])$. Now, we may define the metric $D$ on $L(\mathbb{R})$ by means of the Hausdorff metric $d$ as follows

$$
D(u, v)=\sup _{\alpha \in[0,1]} d\left([u]_{\alpha},[v]_{\alpha}\right)=\sup _{\alpha \in[0 ; 1]} \max \left\{\left|u^{-}(\alpha)-v^{-}(\alpha)\right|,\left|u^{+}(\alpha)-v^{+}(\alpha)\right|\right\}
$$

and

$$
D(u, 0)=\sup _{\alpha \in[0 ; 1]} \max \left\{\left|u^{-}(\alpha)\right|,\left|u^{+}(\alpha)\right|\right\}=\max \left\{\left|u^{-}(\alpha)\right|,\left|u^{+}(\alpha)\right|\right\} .
$$

Let $f, g:[a, b] \rightarrow L(\mathbb{R})$, be fuzzy number valued functions. The parametric representation is as follows: $[f(x)]^{r}=\left[f_{-}^{(r)}(x), f_{+}^{(r)}(x)\right]$, for every $x \in[a, b]$ and every $r \in[0,1]$. Then, the distance between $f$ and $g$ is given by

$$
D^{*}(f, g)=\sup _{x \in[a, b]} \sup _{r \in[0,1]} \max \left\{\left|f_{-}^{(r)}-g_{-}^{(r)}\right|,\left|f_{+}^{(r)}-g_{+}^{(r)}\right|\right\} .
$$

Fuzzy function $f:[a, b] \rightarrow L(\mathbb{R})$, is continuous at $x_{0} \in[a, b]$ if for each $\varepsilon>0$ there is a $\delta>0$ such that $D\left(f(x), f\left(x_{0}\right)\right)<\varepsilon$, whenever $x \in[a, b]$ with $\left|x-x_{0}\right|<\delta$. If $f$ is continuous in each point on $[a, b]$, then we say that it is continuous whole $[a, b]$. The class of continuous function we will denote by $C_{F}[a, b]$.

A sequence $u=\left(u_{k}\right)$ of fuzzy numbers is a function $u$ from the set $\mathbb{N}$, into the set $L(\mathbb{R})$. The fuzzy number $u_{k}$ denotes the value of the function at $k \in \mathbb{N}$ and is called as the $k$-th term of the sequence. By $w(F)$, we denote the set of all sequences of fuzzy numbers. A sequence $\left(u_{n}\right) \in w(F)$ is said to be convergent to $u \in L(\mathbb{R})$, if for every $\varepsilon>0$ there exists an $n_{0}=n_{0}(\varepsilon) \in \mathbb{N}$ such that

$$
D\left(u_{n}, u\right)<\varepsilon \text { for all } n>n_{0} .
$$

Definition 2.1. Let $X=\left(X_{k}\right)$ be a sequence of fuzzy numbers. The sequence $X$ is said to converge weighted statistically to a fuzzy number $X_{0}$, if for every $\varepsilon>0$

$$
\lim _{n \rightarrow \infty} \frac{1}{(n+1)(n+2)}\left|\left\{k \leq(n+1)(n+2): D\left(X_{k}, X_{0}\right) \geq \varepsilon\right\}\right|=0 .
$$

The above type of convergence will be denoted as

$$
s t_{F}-\lim _{n} X_{n}=X_{0} .
$$


Definition 2.2. Let $X=\left(X_{k}\right)$ be a sequence of fuzzy numbers. The sequence $X$ is said to be statistically Cesáro second order summable to a fuzzy number $X_{0}$ if the sequence

$$
(C, 2)_{n}(X)=\frac{1}{(n+1)(n+2)} \sum_{k=0}^{n}(n+1-k) X_{k},
$$

is statistically convergent to $X_{0}$, where the sum in $(C, 2)_{n}(X)$ is usual addition of fuzzy real numbers through $\alpha$ - level sets. That is $\left(X_{k}\right)$ is statistically Cesáro second order summable to the fuzzy number $X_{0}$, if for every $\varepsilon>0$

$$
\lim _{n \rightarrow \infty} \frac{1}{(n+1)(n+2)}\left|\left\{k \leq(n+1)(n+2): D\left((C, 2)_{n}, X_{0}\right) \geq \varepsilon\right\}\right|=0 .
$$

The above type of convergence will be denoted as

$$
s t_{(C, 2)}-\lim _{n} X_{n}=X .
$$

\section{Statistical fuzzy Korovkin type theorem}

Let us denote by $C[a, b]$ the space of continuous function defined in the $[a, b]$. As we know, this space equipped with supremum norm

$$
\|f\|=\sup _{x \in[a, b]}|f(x)|
$$

is a complete metric space.

In this section we prove fuzzy Korovkin type theorem via the concept of statistical summability $(C, 2)$. Let $f:[a, b] \rightarrow L(R)$ be fuzzy number valued functions. Then $f$ is said to be fuzzy continuous at $x_{0} \in[a, b]$ provided that whenever $x_{n} \rightarrow x_{0}$, then $D\left(f\left(x_{n}\right), f\left(x_{0}\right)\right) \rightarrow \infty$ as $n \rightarrow \infty$. Also, we say that $f$ is fuzzy continuous on $[a, b]$ if it is fuzzy continuous at every point $x \in[a, b]$. The set of all fuzzy continuous functions on the interval $[a, b]$ is denoted by $C_{F}[a, b]$ (see, for instance, [3]).

Let $L: C_{F}[a, b] \rightarrow C_{F}[a, b]$ be an operator. Then $L$ is said to be fuzzy linear, if for every $\alpha, \beta \in \mathbb{R}$, any $f, g \in C_{F}[a, b]$ and for every $x \in[a, b]$,

$$
L(\alpha f+\beta g ; x)=\alpha L(f ; x)+\beta L(g ; x),
$$

holds. $L$ is said to be fuzzy positive linear operator if it is fuzzy linear and the condition $L(f ; x) \preceq L(g ; x)$ is satisfied for any $f, g \in C_{F}[a, b]$ and for all $x \in[a, b]$ with $f(x) \preceq$ $g(x)$. Last relation is fulfilled if and only if $f_{-}^{(r)}(x) \leq g_{-}^{(r)}(x)$ and $f_{+}^{(r)}(x) \leq g_{+}^{(r)}(x)$, where $[f(x)]^{(r)}=\left[f_{-}^{(r)}(x), f_{+}^{(r)}(x)\right]$. The fuzzy Korovkin type theorem was investigated by many authors(see $[3,4,2])$ and statistical version of the theorem, was given by [4], as follows.

Theorem 3.1. ([3]) Let $\left\{L_{n}\right\}_{n \in \mathbb{N}}: C_{F}[a, b] \rightarrow C_{F}[a, b]$, be a sequence of fuzzy positive linear operators. Assume that there exists a corresponding sequence $\left\{{\tilde{L_{n}}}_{n \in \mathbb{N}}\right.$ : $C[a, b] \rightarrow C[a, b]$, of linear positive operators, with the property:

$$
\left\{L_{n}(f ; x)\right\}_{ \pm}^{(r)}=\tilde{L_{n}}\left(f_{ \pm}^{(r)} ; x\right)
$$


for all $x \in[a, b], r \in[0,1], n \in \mathbb{N}$ and $f \in C_{F}[a, b]$. Also assume that

$$
\lim _{n \rightarrow \infty}\left\|\tilde{L_{n}}\left(e_{i}\right)-e_{i}\right\|=0, \quad \text { for each } i=0,1,2,
$$

where $e_{i}=x^{i}$. Then, for all $f \in C_{F}[a, b]$, we have

$$
\lim _{n} D^{*}\left(L_{n}(f), f\right)=0 .
$$

Later one, this result is extended to summability matrix as follows

Theorem 3.2. ([4]) Let $A=\left(a_{j_{n}}\right)$ be a non-negative regular summability method matrix and let $\left\{L_{n}\right\}_{n \in \mathbb{N}}$ be a sequence of fuzzy positive linear operators from $C_{F}[a, b]$ into itself. Assume that there exists a corresponding sequence $\left\{\tilde{L_{n}}\right\}_{n \in \mathbb{N}}$ of positive linear operators from $C[a, b]$ into itself with the property (3.1). Assume further that

$$
s t_{A}-\lim _{n \rightarrow \infty}\left\|\tilde{L_{n}}\left(e_{i}\right)-e_{i}\right\|=0, \quad \text { for each } i=0,1,2,
$$

where $e_{i}=x^{i}$. Then, for all $f \in C_{F}[a, b]$, we have

$$
s t_{A}-\lim _{n} D^{*}\left(L_{n}(f), f\right)=0 .
$$

Now we prove the fuzzy Korovkin type theorem for statistical convergence, using the notion of the statistical summability method $(C, 2)$.

Theorem 3.3. Let $\left\{L_{n}\right\}_{n \in \mathbb{N}}$ be a sequence of fuzzy positive linear operators from $C_{F}[a, b]$ into itself. Assume that there exists a corresponding sequence $\left\{{\tilde{L_{n}}}_{n} \in \mathbb{N}\right.$ of positive linear operators from $C[a, b]$ into itself with the property (3.1). Also assume that

$$
s t_{(C, 2)}-\lim _{n \rightarrow \infty}\left\|\tilde{L_{n}}\left(e_{i}\right)-e_{i}\right\|=0, \quad \text { for each } i=0,1,2,
$$

where $e_{i}=x^{i}$. Then, for all $f \in C_{F}[a, b]$, we have

$$
s t_{(C, 2)}-\lim _{n} D^{*}\left(L_{n}(f), f\right)=0 .
$$

Proof. Let $f \in C_{F}[a, b]$ for $x \in[a, b]$ and $r \in[0,1]$. By hypothesis $f_{ \pm}^{(r)} \in C[a, b]$, which means that for every $\varepsilon>0$ there exists a $\delta(\varepsilon)>0$, and for any $y \in[a, b]$ such that $|x-y|<\delta$ we obtain $\left|f_{ \pm}^{(r)}(x)-f_{ \pm}^{(r)}(y)\right|<\varepsilon$. From last relation and boundedness of function $f_{ \pm}^{(r)}(x)$, we get

$$
\left|f_{ \pm}^{(r)}(x)-f_{ \pm}^{(r)}(y)\right| \leq \varepsilon+2\left\|f_{ \pm}^{(r)}\right\| \frac{(x-y)^{2}}{\delta^{2}} .
$$

Considering linearity and positivity of the operators $\tilde{L_{n}}$, we have for each $n \in \mathbb{N}$, that

$$
\begin{gathered}
\left|\tilde{L_{n}}\left(f_{ \pm}^{(r)} ; x\right)-f_{ \pm}^{(r)}(x)\right| \leq\left|\tilde{L_{n}}\left(\left|f_{ \pm}^{(r)}(x)-f_{ \pm}^{(r)}(y)\right| ; x\right)\right|+|| f_{ \pm}^{(r)}||\left|\tilde{L_{n}}\left(e_{0} ; x\right)-e_{0}(x)\right| \leq \varepsilon \\
\quad+\left(\varepsilon+\left\|f_{ \pm}^{(r)}\right\|\right) \cdot\left|\tilde{L_{n}}\left(e_{0} ; x\right)-e_{0}(x)\right|+\frac{2\left\|f_{ \pm}^{(r)}\right\|}{\delta^{2}}\left|\tilde{L_{n}}\left((x-y)^{2} ; x\right)\right|,
\end{gathered}
$$

if we put $M=\max \{|a|,|b|\}$, we have

$$
\left|\tilde{L_{n}}\left(f_{ \pm}^{(r)} ; x\right)-f_{ \pm}^{(r)}(x)\right| \leq \varepsilon+\left(\varepsilon+\left\|f_{ \pm}^{(r)}\right\|+\frac{2 x^{2}}{\delta^{2}}\left\|f_{ \pm}^{(r)}\right\|\right) \cdot\left|\tilde{L_{n}}\left(e_{0} ; x\right)-e_{0}(x)\right|
$$




$$
\begin{gathered}
+\frac{4 x}{\delta^{2}}\left\|f_{ \pm}^{(r)}\right\|\left|\tilde{L_{n}}\left(e_{1} ; x\right)-e_{1}(x)\right|+\frac{2}{\delta^{2}}\left\|f_{ \pm}^{(r)}\right\|\left|\tilde{L_{n}}\left(e_{2} ; x\right)-e_{2}(x)\right| \leq \\
\left|\tilde{L_{n}}\left(f_{ \pm}^{(r)} ; x\right)-f_{ \pm}^{(r)}(x)\right| \leq \varepsilon+\left(\varepsilon+\left\|f_{ \pm}^{(r)}\right\|+\frac{2 M^{2}}{\delta^{2}}\left\|f_{ \pm}^{(r)}\right\|\right) \cdot\left|\tilde{L_{n}}\left(e_{0} ; x\right)-e_{0}(x)\right| \\
+\frac{4 M}{\delta^{2}}\left\|f_{ \pm}^{(r)}\right\|\left|\tilde{L_{n}}\left(e_{1} ; x\right)-e_{1}(x)\right|+\frac{2}{\delta^{2}}\left\|f_{ \pm}^{(r)}\right\|\left|\tilde{L_{n}}\left(e_{2} ; x\right)-e_{2}(x)\right| .
\end{gathered}
$$

Let

$$
M_{ \pm}^{(r)}(\varepsilon)=\max \left\{\varepsilon+\left\|f_{ \pm}^{(r)}\right\|+\frac{2 M^{2}}{\delta^{2}}\left\|f_{ \pm}^{(r)}\right\|, \frac{4 M}{\delta^{2}}\left\|f_{ \pm}^{(r)}\right\|, \frac{2}{\delta^{2}}\left\|f_{ \pm}^{(r)}\right\|\right\} .
$$

Taking supremum on the above inequality for $x \in[a, b]$, we obtain

$$
\left\|\tilde{L_{n}}\left(f_{ \pm}^{(r)}\right)-f_{ \pm}^{(r)}\right\| \leq \varepsilon+M_{ \pm}^{(r)}(\varepsilon)\left\{\left\|\tilde{L_{n}}\left(e_{0}\right)-e_{0}\right\|+\left\|\tilde{L_{n}}\left(e_{1}\right)-e_{1}\right\|+\left\|\tilde{L_{n}}\left(e_{2}\right)-e_{2}\right\|\right\} .
$$

Now using into consideration relation (3.1) and relation (3.4), we have

$$
\begin{gathered}
D^{*}(f, g)=\sup _{x \in[a, b]} D\left(L_{n}(f ; x), f(x)\right) \\
=\sup _{x \in[a, b]} \sup _{r \in[0,1]} \max \left\{\left|\tilde{L_{n}}\left(f_{-}^{(r)} ; x\right)-f_{-}^{(r)}(x)\right|,\left|\tilde{L_{n}}\left(f_{+}^{(r)} ; x\right)-f_{+}^{(r)}(x)\right|\right\} \\
=\sup _{r \in[0,1]} \max \left\{\left\|\tilde{L_{n}}\left(f_{-}^{(r)}\right)-f_{-}^{(r)}\right\|,\left\|\tilde{L_{n}}\left(f_{+}^{(r)}\right)-f_{+}^{(r)}\right\|\right\} .
\end{gathered}
$$

From relations (3.4) and (3.5), it yields

$$
D^{*}\left(L_{n}(f), f\right) \leq \varepsilon+M(\varepsilon)\left\{\left\|\tilde{L_{n}}\left(e_{0}\right)-e_{0}\right\|+\left\|\tilde{L_{n}}\left(e_{1}\right)-e_{1}\right\|+\left\|\tilde{L_{n}}\left(e_{2}\right)-e_{2}\right\|\right\},
$$

where $M(\varepsilon)=\sup _{0 \leq r \leq 1} \max \left\{M_{-}^{(r)}(\varepsilon), M_{+}^{(r)}(\varepsilon)\right\}$.

Let $\varepsilon_{1}>0$, we can choose $0<\varepsilon<\varepsilon_{1}$, and define sets:

$$
\begin{gathered}
A=\left\{n \in \mathbb{N}: D^{*}\left(L_{n}(f), f\right) \geq \varepsilon_{1}\right\}, \\
A_{1}=\left\{n \in \mathbb{N}:\left\|\tilde{L_{n}}\left(e_{0}\right)-e_{0}\right\| \geq \frac{\varepsilon_{1}-\varepsilon}{3 M(\varepsilon)}\right\}, \\
A_{2}=\left\{n \in \mathbb{N}:\left\|\tilde{L_{n}}\left(e_{1}\right)-e_{1}\right\| \geq \frac{\varepsilon_{1}-\varepsilon}{3 M(\varepsilon)}\right\}, \\
A_{3}=\left\{n \in \mathbb{N}:\left\|\tilde{L_{n}}\left(e_{2}\right)-e_{2}\right\| \geq \frac{\varepsilon_{1}-\varepsilon}{3 M(\varepsilon)}\right\} .
\end{gathered}
$$

Then from relation $(3.6)$, we have

$$
A \subset A_{1} \cup A_{2} \cup A_{3} .
$$

Now from last relation and relations (3.2), we get relation (3.3).

Remark 3.4. Our theorem is generalization of the result given in theorem 3.1 and theorem 3.2 , as it is shown on this. 
Example 3.5. Take $A=(C, 2)=\left(c_{j n}\right)$, the Cesáro second order matrix and define the following sequence

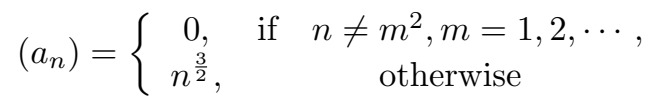

If we use into consideration the fuzzy Bernstein-type operators

$$
B_{n}^{F}(f ; x)=a_{n} \odot \bigoplus_{k=0}^{n}\left(\begin{array}{l}
n \\
k
\end{array}\right) x^{k}(1-x)^{n-k} \odot f_{ \pm}^{(r)}\left(\frac{k}{n}\right)
$$

where $f \in C_{F}[0,1], x \in[0,1]$ and $n \in \mathbb{N}$. We can define

$$
\left\{B_{n}^{F}(f ; x)\right\}_{ \pm}^{(r)}=\tilde{B}_{n}\left(f_{ \pm}^{(r)} ; x\right)=a_{n} \sum_{k=0}^{n}\left(\begin{array}{l}
n \\
k
\end{array}\right) x^{k}(1-x)^{n-k} f_{ \pm}^{(r)}\left(\frac{k}{n}\right)
$$

$f_{ \pm}^{(r)} \in C[0,1]$

Let us define the following operators

$$
L_{n}(f ; x)=\left(1+a_{n}\right) \tilde{B}_{n}(f ; x) .
$$

Then we have:

$$
\begin{gathered}
L_{n}\left(e_{0} ; x\right)=\left(1+a_{n}\right), \\
L_{n}\left(e_{1} ; x\right)=x\left(1+a_{n}\right), \\
L_{n}\left(e_{2} ; x\right)=\left(x^{2}+\frac{x(1-x)}{n}\right)\left(1+a_{n}\right) .
\end{gathered}
$$

The limit $s t_{(C, 2)}-\lim a_{n}$, exist and it is:

$$
\sum_{n:\left|a_{n}-0\right| \geq \varepsilon} c_{j n}=\sum_{n:\left|a_{n}-0\right| \geq \varepsilon} \frac{1}{(j+1)(j+2)} \leq \frac{j^{\frac{3}{2}}}{(j+1)(j+2)} \rightarrow 0, \quad \text { as } \quad j \rightarrow \infty,
$$

which means that $s t_{(C, 2)}-\lim a_{n}=0$.

From above relation we get

$$
s t_{(C, 2)}-\lim _{n \rightarrow \infty}\left\|L_{n}\left(e_{i}\right)-e_{i}\right\|=0, \quad \text { for each } i=0,1,2
$$

and from theorem 3.3, we obtain

$$
s t_{(C, 2)}-\lim _{n} D^{*}\left(L_{n}(f), f\right)=0 .
$$

However, $\left(a_{n}\right)$ is not convergent in usual sense, the sequence $\left\{B_{n}^{F}(f ; x)\right\}$ is not fuzzy convergent to $f$. 


\section{Statistical fuzzy rate of convergence}

In this section, we investigate the rate of the Cesáro second order operators, statistical convergence of positive linear operators in the space $C_{F}[a, b]$.

Definition 4.1. Let $\left(a_{n}\right)$ be any nondecreasing sequence of positive numbers. We say that the sequence of functions $\left(f_{n}\right) \in C_{F}[a, b]$ is Cesáro second order statistical convergent to a function $f$ with the rate of convergence given by $o\left(a_{n}\right)$, if, for each $\varepsilon>0$,

$$
\left.\lim _{n \rightarrow \infty} \frac{1}{(n+1)(n+2) a_{n}} \mid\left\{m \leq(n+1)(n+2) \quad \text { and } \quad D^{*}\left((C, 2)_{m} ; f\right)\right) \geq \varepsilon\right\} \mid=0 .
$$

In this case, we write

$$
f_{n}-f=o\left(a_{n}\right)\left((C, 2)_{n}-s t a t\right) .
$$

Lemma 4.2. Let $\left(a_{n}\right)$ and $\left(b_{n}\right)$ be two nondecreasing sequences of positive numbers. Suppose also that the sequences $\left(f_{n}\right)$ and $\left(g_{n}\right)$ are constrained by

$$
f_{n}-f=o\left(a_{n}\right)\left((C, 2)_{n}-s t a t\right) \quad \text { and } \quad g_{n}-g=o\left(b_{n}\right)\left((C, 2)_{n}-s t a t\right),
$$

respectively. Then

1. $\alpha\left(f_{n}-f\right)=o\left(a_{n}\right)\left((C, 2)_{n}-\right.$ stat $)$ for any scalar $\alpha$;

2. $\left(f_{n}-f\right) \pm\left(g_{n}-g\right)=o\left(c_{n}\right)\left((C, 2)_{n}-\right.$ stat $)$

3. $\left(f_{n}-f\right)\left(g_{n}-g\right)=o\left(a_{n} b_{n}\right)\left((C, 2)_{n}-\right.$ stat $)$,

where

$$
c_{n}:=\max \left\{a_{n}, b_{n}\right\} .
$$
as follows:

Now, by defining the modules of continuity, for a given function $f(x) \in C_{F}[a, b]$,

Definition 4.3. Let $f:[a, b] \rightarrow E$ be a fuzzy real number valued function. We define the modulus of continuity of $f$ by

$$
\omega_{1}^{F}(f, \delta)=\sup _{x, y \in[a, b]} D(f(x), f(y))
$$

for every $|x-y| \leq \delta$ and any $0<\delta \leq b-a$.

We now state and prove the following result.

Theorem 4.4. Let $\left(L_{n}\right)$ be a sequence of fuzzy positive linear operators from $C_{F}[a, b]$ into $C_{F}[a, b]$. Assume that there exists a corresponding sequence $\left\{\tilde{L_{n}}\right\}_{n \in \mathbb{N}}$ of positive linear operators from $C[a, b]$ into itself with the property (3.1). Suppose that $\left(a_{n}\right)$ and $\left(b_{n}\right)$ are non-decreasing sequence and also that the operators $\tilde{L_{n}}$ satisfy the following conditions:

1. $\left\|\tilde{L_{n}}\left(e_{0}\right)-e_{0}\right\|=(\mathrm{C}, 2)_{\mathrm{n}}-$ stat $o\left(a_{n}\right)$ as $n \rightarrow \infty$,

2. $\omega_{1}^{F}\left(f, \lambda_{n}\right)=(\mathrm{C}, 2)_{\mathrm{n}}-$ stat $o\left(b_{n}\right)$ as $n \rightarrow \infty$

where

$$
\lambda_{n}=\sqrt{\| \tilde{L_{n}(\varphi) \|}} \quad \text { and } \quad \varphi_{y}=(y-x)^{2}, \text { for all } \in[a, b]
$$


Then, for all $f \in C_{F}[a, b]$, we have

$$
\left\|\tilde{L_{n}}(f)-f\right\|=(\mathrm{C}, 2)_{\mathrm{n}}-\operatorname{stat} o\left(c_{n}\right) \text {, as } n \rightarrow \infty,
$$

where $c_{n}=\max \left\{a_{n}, b_{n}\right\}$, for each $n \in \mathbb{N}$.

Proof. Let $f \in C_{F}[a, b]$. Then,

$$
\tilde{L_{k}}\left(f_{ \pm}^{(r)}, x\right)-f_{ \pm}^{(r)}(x)=\tilde{L_{k}}\left(f_{ \pm}^{(r)}, x\right)-f_{ \pm}^{(r)}(x) \tilde{L_{k}}(1, x)+f_{ \pm}^{(r)}(x)\left[\tilde{L_{k}}(1, x)-1\right]
$$

and

$$
\left|f_{ \pm}^{(r)}(x)-f_{ \pm}^{(r)}(y)\right| \leq \omega_{1}^{F}(f, \delta)\left(\frac{|x-y|}{\delta}+1\right),
$$

in both cases, where $|x-y| \geq \delta$ and $|x-y| \leq \delta$.

By using the relations (4.1) and (4.2), we get the following estimate:

$$
\begin{gathered}
\left|\tilde{L_{n}}\left(f_{ \pm}^{(r)}, x\right)-f_{ \pm}^{(r)}(x)\right| \leq\left|\tilde{L_{n}}\left(\left|f_{ \pm}^{(r)}(y)-f_{ \pm}^{(r)}(x)\right|, x\right)\right|+\left|\tilde{f_{ \pm}^{(r)}}(x)\right| \cdot\left|\tilde{L_{n}}(1, x)-1\right| \\
\leq \tilde{L_{n}}\left(\frac{|x-y|}{\delta}+1, x\right) \tilde{\omega}_{1}^{F}(f, \delta)+|f(x)| \cdot\left|\tilde{L_{n}}(1, x)-1\right| \text { (by Cauchy-Schwartz inequality) } \\
\leq \frac{1}{\delta} \tilde{L_{n}}\left((x-y)^{2}, x\right)^{\frac{1}{2}} \tilde{L_{n}}(1, x)^{\frac{1}{2}} \omega_{1}^{F}(f, \delta)+\tilde{L_{n}}(1, x) \omega_{1}^{F}(f, \delta) \\
\quad+\left|f_{ \pm}^{(r)}(x)\right| \cdot\left|\tilde{L_{n}}(1, x)-1\right|\left(\text { for } \delta=\lambda_{n},\right. \text { we get) } \\
\leq K\left|\tilde{L_{n}}(1, x)-1\right|+2 \omega_{1}^{F}(f, \delta)+\omega_{1}^{F}(f, \delta)\left|\tilde{L_{n}}(1, x)-1\right| \\
+\omega_{1}^{F}(f, \delta) \sqrt{\left|\tilde{L_{n}}(1, x)-1\right|}
\end{gathered}
$$

where $K=\left\|f_{ \pm}^{(r)}\right\|$. Now, by using relations (1) and (2) in the theorem and lemma 4.2 , we complete proof of Theorem.

\section{Statistical fuzzy Voronovskaya type theorem}

In this section we show positive linear operators

$$
D_{n}(f ; x)=\frac{\left(1+b_{n}\right)}{n^{2}} \tilde{B}_{n}(f ; x),
$$

where sequence $n\left(b_{n}\right)=\left(a_{n}\right)$, and $\left(a_{n}\right)$, is defined in example 3.5 , satisfy a Voronovskaja type property in the $(C, 2)$ - statistically convergence sense. We first prove the following lemma.

Lemma 5.1. For $x \in[a, b]$, and $\Phi(y)=y-x$ then

$$
n^{2} D_{n}\left(\Phi^{4}\right) \sim x^{2}\left(2 x^{2}+1\right)(x-1)((C, 2)-\text { stat. }) \quad \text { on } \quad[a, b] \text {. }
$$


Proof. After some calculations we get:

$$
\begin{gathered}
n^{2} D_{n}\left(\Phi^{4}\right)=\left(1+b_{n}\right)\left[\left(2-\frac{5}{n}+\frac{8}{n^{2}}-\frac{11}{n^{3}}+\frac{6}{n^{4}}\right) x^{5}+\left(-2+\frac{4}{n}-\frac{5}{n^{2}}+\frac{9}{n^{3}}-\frac{6}{n^{4}}\right) x^{4}\right. \\
\left.+\left(1-\frac{2}{n}+\frac{1}{n^{3}}\right) x^{3}-\left(1-\frac{2}{n}+\frac{3}{n^{3}}-\frac{2}{n^{4}}\right) x^{2}+\left(\frac{1}{n^{2}}-\frac{3}{n^{3}}+\frac{2}{n^{4}}\right) x\right] .
\end{gathered}
$$

Thus we obtain:

$$
\begin{gathered}
\left|n^{2} D_{n}\left(\Phi^{4}\right)-x^{2}\left(2 x^{2}+1\right)(x-1)\right| \leq\left|\left(1+b_{n}\right)-1\right|\left|\left(2 x^{5}-2 x^{4}+x^{3}-x^{2}\right)\right| \\
+\left|\left(-\frac{5}{n}+\frac{8}{n^{2}}-\frac{11}{n^{3}}+\frac{6}{n^{4}}\right) x^{5}\right|+\left|\left(\frac{4}{n}-\frac{5}{n^{2}}+\frac{9}{n^{3}}-\frac{6}{n^{4}}\right) x^{4}\right|+\left|\left(-\frac{2}{n}+\frac{1}{n^{3}}\right) x^{3}\right| \\
+\left|\left(-\frac{2}{n}+\frac{3}{n^{3}}-\frac{2}{n^{4}}\right) x^{2}\right|+\left|\left(\frac{1}{n^{2}}-\frac{3}{n^{3}}+\frac{2}{n^{4}}\right) x\right| \rightarrow 0((C, 2)-\text { stat. }),
\end{gathered}
$$

as $n \rightarrow \infty$, on $[a, b]$. This completes proof of the Lemma.

In what follows we establish the following Voronovskaya fuzzy type theorem for operators $D_{n}$, defined as in above Lemma. Before given the main result of this section we will give some concepts related to the $\mathrm{H}$-derivatives for the fuzzy functions.

A function $f:\left[x_{0} ; x_{0}+\alpha\right] \rightarrow R_{F}$, for $\alpha>0$, is $H$-derivative at $x \in T$ if there exists a $f^{\prime}(x) \in R_{F}$ such that the limits

$$
\lim _{h \rightarrow 0^{+}} \frac{f(x+h)-f(x)}{h}, \lim _{h \rightarrow 0^{+}} \frac{f(x)-f(x-h)}{h}
$$

exists and are equal to $f^{\prime}(x)$.

We assume that the $H$ - differences $f(x+h)-f(x), f(x)-f(x-h) \in R_{F}$ in a neighborhood of $x$. We call $f^{\prime}(x)$ the derivative or $H$ - derivative of $f$ at $x$ (for more details see [15]). In paper [2], was given the Taylor formula for fuzzy functions as follows:

Theorem 5.2. Let $T=\left[x_{0}, x_{0}+\alpha\right] \subset \mathbb{R}$, and $\alpha>0$. We assume that $f^{(i)}: T \rightarrow R_{F}$ are $H$ - differentiable for all $i \in\{0,1,2,3, \cdots, n-1\}$, for any $x \in T$. (It means that there exists in $R_{F}$ the $H$ - differences $f^{(i)}(x+h)-f^{(i)}(x), f^{(i)}(x)-f^{(i)}(x-h)$, $i \in\{0,1,2,3 \cdots, n-1\}$ for all $h$ such that $0<h<\alpha$. Furthermore there exists $f^{(i+1)}(x) \in R_{F}$ such that limits in D- metrics exist and

$$
f^{(i+1)}(x)=\lim _{h \rightarrow 0^{+}} \frac{f^{(i)}(x+h)-f^{(i)}(x)}{h}=\lim _{h \rightarrow 0^{+}} \frac{f^{(i)}(x)-f^{(i)}(x-h)}{h},
$$

for all $i \in\{0,1,2,3 \cdots, n-1\}$.) Also we assume that $f^{(n)}$, is fuzzy continuous on $T$. Then for $s \geq a ; s, a \in T$ we obtain

$$
f(s)=f(a)+\frac{f^{\prime}(a)}{1 !}(s-a)+\frac{f^{\prime \prime}(a)}{2 !}(s-a)^{2}+\cdots+\frac{f^{(n-1)}(a)}{(n-1) !}(s-a)^{n-1}+R_{n}(s, a),
$$

where

$$
R_{n}(s, a)=\int_{a}^{s}\left(\int_{a}^{s_{1}} \cdots\left(\int_{a}^{s_{n-1}} f^{(n)}\left(s_{n}\right) d s_{n}\right) d s_{n-1} \cdots\right) d s_{1}
$$


above integration is in sense of Fuzzy-Riemann integral and $R_{n}(s, a)$ is fuzzy continuous on $T$ as a function of $s$.

Theorem 5.3. For every $f:[a, b] \rightarrow R_{F}$, we assume that there exists $f^{\prime}, f^{\prime \prime} \in R_{F}$, then

on $[a, b]$.

$$
n\left[n^{2} D_{n}(f)-f(x)\right] \sim \frac{1}{2}\left(x-x^{2}\right) f^{\prime \prime}(x)((C, 2)-\text { stat. }),
$$

Proof. Let us suppose that $f^{\prime}, f^{\prime \prime} \in R_{F}$ and $x \in[a, b]$. Define

$$
\psi_{x}(y)= \begin{cases}\frac{f(y)-f(x)-(y-x) f^{\prime}(x)-\frac{1}{2}(y-x)^{2} f^{\prime \prime}(x)}{(y-x)^{2}} & \text { for } x \neq y \\ 0 & \text { for } x=y\end{cases}
$$

Then $\psi_{x}(x)=0$ and $\psi_{x} \in C_{F}[a, b]$. By Taylor's formula, we get

$$
f(y)=f(x)+(y-x) f^{\prime}(x)+\frac{1}{2}(y-x)^{2} f^{\prime \prime}(x)+(y-x)^{2} \psi_{x}(y) .
$$

Knowing that

$$
D_{n}(1, x)=\frac{\left(1+b_{n}\right)}{n^{2}} ; D_{n}((y-x), x)=0 \quad \text { and } \quad D_{n}\left((y-x)^{2}, x\right)=\left(1+b_{n}\right) \frac{x-x^{2}}{n^{3}}
$$

and after operating in the both sides of relation (5.1) by operator $D_{n}$, we obtain:

$$
n^{2} D_{n}(f)=f(x)+b_{n} f(x)+\frac{f^{\prime \prime}(x)}{2} \frac{x-x^{2}}{n}\left(1+b_{n}\right)+\left(1+b_{n}\right) D_{n}\left(\Phi^{2} \psi_{x}, x\right),
$$

which yields

$$
\begin{gathered}
\left|n\left[n^{2} D_{n}(f)-f(x)\right]-\frac{f^{\prime \prime}(x)}{2}\left(x-x^{2}\right)\right| \leq \\
n b_{n}|f(x)|+b_{n}\left|\frac{f^{\prime \prime}(x)}{2}\left(x-x^{2}\right)\right|+n\left(1+b_{n}\right)\left|D_{n}\left(\Phi^{2} \psi_{x}, x\right)\right|,
\end{gathered}
$$

respectively

$$
\left|n\left[n^{2} D_{n}(f)-f(x)\right]-\frac{f^{\prime \prime}(x)}{2}\left(x-x^{2}\right)\right| \leq n b_{n} M+n\left|D_{n}\left(\Phi^{2} \psi_{x}, x\right)\right|+n b_{n}\left|D_{n}\left(\Phi^{2} \psi_{x}, x\right)\right|,
$$

where $\Phi(y)=y-x$ and $M=\|f\|_{C_{F}[a, b]}+\left\|f^{\prime \prime}\right\|_{C_{F}[a, b]}$. After application of the Cauchy-Schwartz inequality in the terms of the right side of the relation (5.2), we obtain:

$$
n\left|D_{n}\left(\Phi^{2} \psi_{x}, x\right)\right| \leq\left[n^{2} D_{n}\left(\Phi^{4}, x\right)\right]^{\frac{1}{2}} \cdot\left[D_{n}\left(\psi_{x}^{2}, x\right)\right]^{\frac{1}{2}} .
$$

Putting $\eta_{x}(y)=\left(\psi_{x}(y)\right)^{2}$, we get that $\eta_{x}(x)=0$ and $\eta_{x}(\cdot) \in C_{F}[a, b]$. Also

$$
a_{n}\left|D_{n}\left(\Phi^{2} \psi_{x}, x\right)\right| \leq a_{n}\left[D_{n}\left(\Phi^{4}, x\right)\right]^{\frac{1}{2}} \cdot\left[D_{n}\left(\psi_{x}^{2}, x\right)\right]^{\frac{1}{2}}
$$

where $a_{n} \rightarrow 0\left((C, 2)_{n}-\right.$ stat $)$.

Now from Theorem 3.3, it follows that

$$
D_{n}\left(\eta_{x}\right) \rightarrow 0\left((C, 2)_{n}-s t a t\right)
$$


on $[\mathrm{a}, \mathrm{b}]$. Now, from relations $(5.3),(5.5),(5.4)$ and Lemma 5.1, we have

$$
n\left(1+b_{n}\right) D_{n}\left(\Phi^{2} \psi_{x}, x\right) \rightarrow 0\left((C, 2)_{n}-s t a t\right),
$$

on $[a, b]$. For a given $\varepsilon>0$, we define the following sets:

$$
\begin{gathered}
A_{n}(x, \varepsilon)=\left|\left\{k: k \leq(n+1)(n+2):\left|n\left[n^{2} D_{n}(f)-f(x)\right]-\frac{f^{\prime \prime}(x)}{2}\left(x-x^{2}\right)\right| \geq \varepsilon\right\}\right|, \\
A_{1, n}(x, \varepsilon)=\left|\left\{k: k \leq(n+1)(n+2):\left|k b_{k}\right| \geq \frac{\varepsilon}{2 M}\right\}\right|,
\end{gathered}
$$

and

$$
A_{2, n}(x, \varepsilon)=\left|\left\{k: k \leq(n+1)(n+2):\left|k\left(1+b_{k}\right) D_{k}\left(\Phi^{2} \psi_{x}, x\right)\right| \geq \frac{\varepsilon}{2}\right\}\right| .
$$

From last relation we have

$$
\frac{A_{n}(x, \varepsilon)}{(n+1)(n+2) a_{n}} \leq \frac{A_{1, n}(x, \varepsilon)}{(n+1)(n+2) a_{n}}+\frac{A_{2, n}(x, \varepsilon)}{(n+1)(n+2) a_{n}} .
$$

From definition of the sequence $\left(b_{n}\right)$, we get

$$
n b_{n} \rightarrow 0\left((C, 2)_{n}-s t a t\right)
$$

on $[\mathrm{a}, \mathrm{b}]$. Now from relations (5.6) and (5.8), the right hand side of the relation (5.7), tends to zero as $n \rightarrow \infty$. Therefore, we have

$$
\lim _{n \rightarrow \infty} \frac{A_{n}(x, \varepsilon)}{(n+1)(n+2) a_{n}}=0
$$

which proves that

$$
n\left[n^{2} D_{n}(f)-f(x)\right] \sim \frac{1}{2}\left(x-x^{2}\right) f^{\prime \prime}(x)\left((C, 2)_{n}-s t a t\right)
$$

on $[a, b]$.

\section{Concluding remarks}

In this section, we will give some remarks related to the results obtained in this paper and their relationship with other results.

Remark 6.1. Suppose that we replace the conditions (1) and (2) in Theorem 4.4 by the following condition:

$$
\tilde{L_{n}}\left(x_{i}\right)-x_{i}=o\left(a_{n_{i}}\right)\left((C, 2)_{n}-s t a t\right) \quad \text { on } \quad[a, b](i=0,1,2) .
$$

Then, since

$$
\tilde{L_{n}}\left(\psi^{2} ; x\right)=\tilde{L_{n}}\left(t^{2}, x\right)-2 x \tilde{L_{n}}(t, x)+x^{2} \tilde{L_{n}}(1, x)
$$

we may write

$$
\tilde{L_{n}}\left(\psi^{2}, x\right) \leq K\left[\left|\tilde{L_{n}}(1, x)-1\right|+\left|\tilde{L_{n}}(t, x)-t\right|+\left|\tilde{L_{n}}\left(t^{2}, x\right)-t^{2}\right|\right],
$$

where

$$
K=1+2\|t\|+\left\|t^{2}\right\| .
$$


Now it follows from above relations and Lemma 4.2 that

$$
\delta_{n}=\sqrt{\tilde{L_{n}}\left(\psi^{2}\right)}=o\left(d_{n}\right)\left((C, 2)_{n}-s t a t\right)
$$

on $[a, b]$, where $d_{n}=\min \left\{a_{n_{0}}, a_{n_{1}}, a_{n_{2}}\right\}$. Hence

$$
\omega\left(f, d_{n}\right)=o\left(d_{n}\right)\left((C, 2)_{n}-s t a t\right)
$$

on $[a, b]$. If those conditions which are given here we can use in Theorem 3.3, we can thus see that, for all $f \in C_{F}[a, b]$,

$$
\tilde{L_{n}}(f)-f=o\left(d_{n}\right)\left((C, 2)_{n}-s t a t\right)
$$

on $[a, b]$. Therefore, if we use the condition (6.1) in Theorem 4.4 instead of the conditions (1) and (2), then we obtain the rates of $(C, 2)_{n}-$ stat convergent of the sequence of positive linear operators in Theorem 3.3.

Acknowledgment. Authors would like to thank referees for carefully reading the paper and give comments, which helped us to improve it.

\section{References}

[1] Altin, Y., Mursaleen, M., Altinok, H., Statistical summability $(C, 1)$ for sequences of fuzzy real numbers and a Tauberian theorem, Journal of Intelligent and Fuzzy Systems, 21(2010), 379-384.

[2] Anastassiou, G.A., Rate of convergence of fuzzy neural network operators, univariate case, J. Fuzzy Math., 10(2002), no. 3, 755-780.

[3] Anastassiou, G.A., On basic fuzzy Korovkin theory, Stud. Univ. Babeş-Bolyai Math., 50(2005), 3-10.

[4] Anastassiou, G.A., Duman, O., Statistical fuzzy approximation by fuzzy positive linear operators, Computers and Mathematics with Applications, 55(2008), 573-580.

[5] Bede, B., Gal, S.G., Almost periodic fuzzy number valued functions, Fuzzy Sets and Systems, 147(2004), 385-403.

[6] Braha, N.L., Geometric properties of the second-order Cesáro spaces, Banach J. Math. Anal., 10(2016), no. 1, 1-14.

[7] Braha, N.L., Some weighted equi-statistical convergence and Korovkin type-theorem, Results Math., 70(2016), no. 3-4, 433-446.

[8] Braha, N.L., Loku, V., Srivastava, H.M., $\Lambda^{2}$-weighted statistical convergence and Korovkin and Voronovskaya type theorems, Appl. Math. Comput., 266(2015), 675-686.

[9] Braha, N.L., Srivastava, H.M., Mohiuddine, S.A., A Korovkin's type approximation theorem for periodic functions via the statistical summability of the generalized de la Vallee Poussin mean, Appl. Math. Comput., 228(2014), 162-169.

[10] Fast, H., Sur la convergence statistique, Colloq. Math., 2(1951), 241-244.

[11] Kadak, U., Braha, N.L., Srivastava, H.M., Statistical weighted $\mathcal{B}$-summability and its applications to approximation theorems, Appl. Math. Comput., 302(2017), 80-96.

[12] Loku, V., Braha, N.L., Some weighted statistical convergence and Korovkin type-theorem, J. Inequal. Spec. Funct., 8(2017), no. 3, 139-150.

[13] Matloka, M., Sequence of fuzzy numbers, BUSEFAL, 28(1986), 28-37.

[14] Nanda, S., On sequence of fuzzy numbers, Fuzzy Sets and Systems, 33(1989), 123-126. 
[15] Puri, M.L., Ralescu, D.A., Differentials of fuzzy functions, J. Math. Anal. Appl., 91 (1983), no. 2, 552-558.

[16] Zadeh, L.A., Fuzzy sets, Information and Control, 8(1965), 338-353.

Naim L. Braha

Research Institute Ilirias, www.ilirias.com, pn, Janina, Ferizaj, 70000, Kosova Department of Mathematics and Computer Sciences, University of Prishtina, Avenue Mother Teresa, No-4, Prishtine, 10000, Kosova e-mail: nbraha@yahoo.com

Valdete Loku

University of Applied Sciences Ferizaj, Rr. Rexhep Bislimi, Pn. Ferizaj, 70000, Kosova

(Corresponding author)

e-mail: valdeteloku@gmail.com 